Finney, D. J., Hazlewood, T. and Smith, M. J. (1955). J. gen. Microbiol. 12, 222-225

\title{
Logarithms to Base 2
}

\section{By D. J. FINNEY,* TAMARA HAZLEWOOD AND MARIAN J. SMITH \\ Lecturership in the Design and Analysis of Scientific Experiment, University of Oxford}

Graphical representation of the growth of a bacterial culture is usually best achieved by using a logarithmic scale for whatever measurement of the density of the culture is made. Moreover, as Monod (1949) has pointed out, both diagrams and associated calculations are more readily interpreted if logarithms to base 2 are used instead of common logarithms (i.e. base 10): an increase of 1 unit on the logarithmic scale then corresponds to the equivalent of one division by each of the cells present at the beginning of the interval.

Rather surprisingly, none of the better-known collections of mathematical tables nor any of the usual sources for miscellaneous tables appears to contain logarithms to base 2. Of course, for any particular number, multiplication of its common logarithm by $3.32193\left(=\log _{2} 10\right)$ gives the logarithm to base 2 , but performance of this multiplication for many series of data is tedious and time consuming. As the result of a suggestion from Dr W. E. van Heyningen, the following table has therefore been computed. $\dagger$ Only 3 places of decimals are shown, since graphical work will certainly not permit greater accuracy and even for arithmetical analysis observational data rarely justify the use of more digits; the table is designed for simple rapid use by microbiologists, not for serious mathematical purposes.

The table shows the logarithm of every integer from 100 to 999 . Thus, by direct reading,

$$
\log _{2} 673=9 \cdot 394 \text {. }
$$

If the highest and lowest densities of a series do not differ by more than a factor of 10, there may be no necessity to construct any logarithms outside the range of the table. For example, if the densities range from 1.7 to 8.9 , expression of density in terms of a volume unit 100 times that of the standard will give values ranging from 170 to 890 ; all logarithms can then be read directly from the table and graphed as they stand.

If the densities extend over a wider range, logarithms of numbers not in the table will be needed. These are obtained by the usual rule that the logarithm of a product or quotient is the sum or difference of the logarithms of the two parts. Thus

$$
\begin{aligned}
\log _{2} 2692 & =\log _{2} 4+\log _{2} 673 \\
& =2 \cdot 000+9 \cdot 394=11 \cdot 394 \\
\log _{2} 0 \cdot 075 & =\log _{2} 300-\log _{2} 400-\log _{2} 10 \\
& =8 \cdot 229-8 \cdot 644-3 \cdot 322=-3 \cdot 737
\end{aligned}
$$

* Present address: University of Aberdeen.

$\dagger$ The table was obtained by multiplication of appropriate entries in the U.S. National Bureau of Standards Table of Natural Logarithms by $\log _{2} e$ and independently checked by multiplication of entries in Chamber's Mathematical Tables (Seven Figure Logarithms) by $\log _{2} 10$. 
When suitable factors are not obvious, powers of 10 can be used, remembering that $\log _{2} 10=3.3219, \log _{2} 100=6 \cdot 6439, \log _{2} 1000=9.9658$; logarithms of powers of 10 up to $10^{10}$ are appended to the table. Hence

$$
\begin{aligned}
\log _{2} 0 \cdot 875 & =\log _{2} 875-\log _{2} 1000 \\
& =9 \cdot 773-9 \cdot 966=-0 \cdot 193 .
\end{aligned}
$$

(Since $0 \cdot 875=7 / 8$, the same value can be obtained as $\log _{2} 700-\log _{2} 800$.) The measurements will rarely be accurate to more than 3 significant digits, but, if required, logarithms of quantities intermediate between tabular values can be derived by the common-sense application of 'linear interpolation'; thus $\log _{2} 2692$ can alternatively be calculated as at $2 / 10$ of the distance between

and

$$
\begin{aligned}
& \log _{2} 2690=8 \cdot 071+3 \cdot 322=11 \cdot 393, \\
& \log _{2} 2700=8 \cdot 077+3 \cdot 322=11 \cdot 399,
\end{aligned}
$$

and is $11 \cdot 394$ as before.

\section{REFERENCE}

Monod, J. (1949). The growth of bacterial cultures. Annu. Rev. Microbiol. 3, 371.

(Received 13 September 1954) 
Logarithms to base 2

\begin{tabular}{|c|c|c|c|c|c|c|c|c|c|c|}
\hline & 0 & 1 & 2 & $\mathbf{3}$ & 4 & 5 & 6. & 7 & 8 & $\theta$ \\
\hline 100 & $6 \cdot 644$ & $6 \cdot 658$ & $6 \cdot 672$ & 6.687 & $6 \cdot 700$ & 6.714 & $6 \cdot 728$ & $6 \cdot 741$ & 6.755 & $6 \cdot 768$ \\
\hline 110 & $\cdot 781$ & $\cdot 794$ & $\cdot 807$ & $\cdot 820$ & $\cdot 833$ & $\cdot 84.5$ & $\cdot 858$ & $\cdot 870$ & $\cdot 883$ & $\cdot 895$ \\
\hline 120 & .907 & .919 & .931 & .943 & .954 & .966 & .977 & .989 & $7 \cdot 000$ & $7 \cdot 011$ \\
\hline 130 & $7 \cdot 022$ & $7 \cdot 033$ & $7 \cdot 044$ & $7 \cdot 055$ & $7 \cdot 066$ & $7 \cdot 077$ & $7 \cdot 087$ & $7 \cdot 098$ & $\cdot 109$ & $\cdot 119$ \\
\hline 140 & $\cdot 129$ & $\cdot 140$ & $\cdot 150$ & $\cdot 160$ & $\cdot 170$ & $\cdot 180$ & $\cdot 190$ & $\cdot 200$ & $\cdot 209$ & $\cdot 219$ \\
\hline 150 & $7 \cdot 229$ & $7 \cdot 238$ & $7 \cdot \mathbf{2 4 8}$ & $7 \cdot 257$ & $7 \cdot 267$ & $7 \cdot 276$ & $7 \cdot 285$ & $7 \cdot 295$ & $7 \cdot 304$ & $7 \cdot 313$ \\
\hline 160 & $\cdot \mathbf{3 2 2}$ & $\cdot 331$ & $\cdot \mathbf{3 4 0}$ & $\cdot 349$ & $\cdot 358$ & •366 & -375 & $\cdot 384$ & •392 & -401 \\
\hline 170 & .409 & .418 & .426 & .435 & .443 & .451 & .459 & .468 & .476 & .484 \\
\hline 180 & .492 & .500 & .508 & .516 & .524 & .531 & .539 & .547 & .555 & $\cdot 562$ \\
\hline 190 & $\cdot 570$ & .577 & .585 & $\cdot 592$ & .600 & $\cdot 607$ & .615 & $\cdot 622$ & $\cdot 629$ & $\cdot 637$ \\
\hline 200 & $7 \cdot 644$ & $7 \cdot 651$ & $7 \cdot 658$ & $7 \cdot 665$ & $7 \cdot 672$ & $7 \cdot 679$ & $7 \cdot 687$ & $7 \cdot 693$ & $7 \cdot 700$ & $7 \cdot 707$ \\
\hline 210 & $\cdot 714$ & .721 & $\cdot 728$ & .735 & $\cdot 741$ & $\cdot 748$ & $\cdot 755$ & $\cdot 762$ & .768 & .775 \\
\hline 220 & .781 & .788 & .794 & .801 & .807 & .814 & .820 & .827 & .833 & .839 \\
\hline 230 & $\cdot 845$ & $\cdot 852$ & $\cdot 858$ & .864 & $\cdot 870$ & .877 & $\cdot 883$ & .889 & .895 & .901 \\
\hline 240 & .907 & .913 & .919 & .925 & .931 & .937 & .943 & .948 & .954 & .960 \\
\hline 250 & $7 \cdot 966$ & 7.972 & $7 \cdot 977$ & $7 \cdot 983$ & $7 \cdot 989$ & $7 \cdot 994$ & 8.000 & $8 \cdot 006$ & 8.011 & 8.017 \\
\hline 260 & $8 \cdot 022$ & 8.028 & $8 \cdot 033$ & 8.039 & $8 \cdot 044$ & 8.050 & .055 & .061 & .066 & .071 \\
\hline 270 & $\cdot 077$ & $\cdot 082$ & $\cdot 087$ & .093 & -098 & $\cdot 103$ & $\cdot 109$ & .114 & $\cdot 119$ & $\cdot 124$ \\
\hline 280 & $\cdot 129$ & $\cdot 134$ & $\cdot 140$ & $\cdot 145$ & $\cdot 150$ & $\cdot 155$ & $\cdot 160$ & $\cdot 165$ & $\cdot 170$ & $\cdot 175$ \\
\hline 290 & $\cdot 180$ & $\cdot 185$ & $\cdot 190$ & $\cdot 195$ & $\cdot 200$ & .205 & .209 & $\cdot 214$ & .219 & .224 \\
\hline 300 & $8 \cdot 229$ & $8 \cdot 234$ & $8 \cdot 238$ & $8 \cdot 243$ & $8 \cdot 248$ & 8.253 & $8 \cdot 257$ & $8 \cdot 262$ & $8 \cdot 267$ & 8.271 \\
\hline 310 & $\cdot 276$ & $\cdot 281$ & $\cdot 285$ & $\cdot 290$ & $\cdot 295$ & .299 & .304 & $\cdot 308$ & .313 & .817 \\
\hline 320 & $\cdot 322$ & $\cdot 326$ & .331 & $\cdot 335$ & $\cdot 340$ & $\cdot 344$ & $\cdot \mathbf{3 4 9}$ &. $\mathbf{3 5 3}$ & $\cdot \mathbf{3 5 8}$ & $\cdot \mathbf{3 6 2}$ \\
\hline 330 & $\cdot 366$ & $\cdot \mathbf{3 7 1}$ & $\cdot 375$ & $\cdot 379$ & .384 & .388 & .392 & .397 & .401 & .405 \\
\hline 340 & .409 & .414 & .418 & $\cdot 422$ & .426 & .430 & .435 & .439 & .443 & .447 \\
\hline 350 & 8.451 & 8.455 & $8 \cdot 459$ & 8.464 & $8 \cdot 468$ & 8.472 & $8 \cdot 476$ & 8.480 & 8.484 & $8 \cdot 488$ \\
\hline 360 & $\cdot 492$ & .496 & $\cdot 500$ & .504 & $\cdot 508$ & .512 & $\cdot 516$ & $\cdot 520$ & .524 & $\cdot 527$ \\
\hline $\mathbf{3 7 0}$ & $\cdot 531$ & $\cdot 535$ & $\cdot 539$ & $\cdot 543$ & $\cdot 547$ & $\cdot 551$ & $\cdot 555$ & -558 & $\cdot 562$ & $\cdot 566$ \\
\hline 380 & $\cdot 570$ & .574 & .577 & $\cdot 581$ & $\cdot 585$ & .589 & .592 & .596 & .600 & $\cdot 604$ \\
\hline 390 & .607 & $\cdot 611$ & .615 & .618 & .622 & .626 & .629 & $\cdot 633$ & .637 & $\cdot 640$ \\
\hline 400 & $8 \cdot 644$ & $8 \cdot 647$ & $8 \cdot 651$ & $8 \cdot 655$ & $8 \cdot 658$ & $8 \cdot 662$ & $8 \cdot 665$ & $8 \cdot 669$ & $8 \cdot 672$ & 8.676 \\
\hline 410 & $\cdot 679$ & $\cdot 683$ & $\cdot 687$ & $\cdot 690$ & $\cdot 693$ & $\cdot 697$ & $\cdot 700$ & $\cdot 704$ & .707 & $\cdot 711$ \\
\hline 4,20 & .714 & $\cdot 718$ & .721 & .725 & .728 & .731 & .735 & .738 & .741 & .745 \\
\hline 430 & .748 & .752 & .755 & .758 & .762 & .765 & $\cdot 768$ & .771 & .775 & $\cdot 778$ \\
\hline 440 & .781 & .785 & .788 & .791 & $\cdot 794$ & .798 & .801 & .804 & .807 & $\cdot 811$ \\
\hline 450 & $8 \cdot 814$ & $8 \cdot 817$ & $8 \cdot 820$ & $8 \cdot 823$ & 8.827 & $8 \cdot 830$ & 8.833 & $8 \cdot 836$ & 8.839 & 8.842 \\
\hline 460 & $\cdot 845$ & $\cdot 849$ & $\cdot 852$ & $\cdot 855$ & $\cdot 858$ & $\cdot 861$ & $\cdot 864$ & .867 & $\cdot 870$ & $\cdot 873$ \\
\hline 470 & .877 & .880 & $\cdot 883$ & .886 & $\cdot 889$ & .892 & .895 & .898 & .901 & .904 \\
\hline 480 & .907 & .910 & .913 & .916 & .919 & .922 & .925 & .928 & .931 & .934 \\
\hline 490 & .937 & .940 & .943 & .945 & .948 & .951 & .954 & .957 & .960 & .963 \\
\hline 500 & 8.966 & 8.969 & 8.972 & 8.974 & 8.977 & 8.980 & 8.983 & 8.986 & 8.989 & 8.992 \\
\hline 510 & .994 & .997 & $9 \cdot 000$ & 9.003 & $9 \cdot 006$ & $9 \cdot 008$ & 9.011 & $9 \cdot 014$ & 9.017 & $9 \cdot 020$ \\
\hline $\mathbf{5 2 0}$ & $9 \cdot 022$ & $9 \cdot 025$ & $\cdot 028$ & $\cdot 031$ & .033 & $\cdot 036$ & $\cdot 039$ & .042 & .044 & .047 \\
\hline 530 & $\cdot 050$ & $\cdot 053$ & .055 & .058 & .061 & .063 & .066 & .069 & .071 & $\cdot 074$ \\
\hline 540 & .077 & .079 & .082 & .085 & .087 & .090 & .093 & .085 & .098 & $\cdot 101$ \\
\hline
\end{tabular}


Logarithms to base 2

\begin{tabular}{|c|c|c|c|c|c|c|c|c|c|c|}
\hline & o & 1 & 2 & 3 & 4 & 5 & 6 & 7 & 8 & 9 \\
\hline 550 & $9 \cdot 103$ & $9 \cdot 106$ & $9 \cdot 109$ & $9 \cdot 111$ & $9 \cdot 114$ & $9 \cdot 116$ & $9 \cdot 119$ & $9 \cdot 122$ & $9 \cdot 124$ & $9 \cdot 127$ \\
\hline 560 & $\cdot 129$ & $\cdot 132$ & $\cdot 134$ & $\cdot 137$ & $\cdot 140$ & $\cdot 142$ & $\cdot 145$ & $\cdot 147$ & $\cdot 150$ & $\cdot 152$ \\
\hline 570 & $\cdot 155$ & $\cdot 157$ & $\cdot 160$ & $\cdot 162$ & $\cdot 165$ & $\cdot 167$ & $\cdot 170$ & $\cdot 172$ & $\cdot 175$ & $\cdot 177$ \\
\hline 580 & $\cdot 180$ & $\cdot 182$ & $\cdot 185$ & $\cdot 187$ & ·190 & $\cdot 192$ & $\cdot 195$ & $\cdot 197$ & $\cdot 200$ & $\cdot 202$ \\
\hline 590 & .205 & .207 & .210 & .212 & .214 & .217 & .219 & $\cdot 222$ & .224 & $\cdot 226$ \\
\hline 600 & $9 \cdot 229$ & $9 \cdot 231$ & $9 \cdot 234$ & $9 \cdot 236$ & $9 \cdot 238$ & $9 \cdot 241$ & $9 \cdot 243$ & $9 \cdot 246$ & $9 \cdot 248$ & $9 \cdot 250$ \\
\hline 610 & $\cdot 253$ & $\cdot 255$ & .257 & .260 & $\cdot 262$ & $\cdot 264$ & .267 & .269 & .271 & .274 \\
\hline 620 & .276 & .278 & .281 & .283 & .285 & .288 & $\cdot 290$ & .292 & .295 & .297 \\
\hline 630 & $\cdot 299$ & $\cdot 301$ & .304 & .306 & .308 & $\cdot \mathbf{3 1 1}$ & .313 & .315 & .317 & $\cdot 320$ \\
\hline 640 & .322 & $\cdot 324$ & .326 & .329 & .331 & $\cdot \mathbf{3 3 3}$ & $\cdot 335$ & .338 & .340 & $\cdot 342$ \\
\hline 650 & $9 \cdot 344$ & $9 \cdot 347$ & $9 \cdot 349$ & $9 \cdot 351$ & 9.353 & 9.355 & 9·358 & $\mathbf{9 \cdot 3 6 0}$ & $9 \cdot 362$ & $9 \cdot 364$ \\
\hline 660 & $\cdot 366$ & $\cdot 369$ & $\cdot 371$ & $\cdot \mathbf{3 7 3}$ & .375 & $\cdot 377$ & $\cdot 379$ & •382 & $\cdot \mathbf{3 8 4}$ & $\cdot 386$ \\
\hline 670 & $\cdot 388$ & .390 & .392 & .394 & $\cdot 397$ & .399 & .401 & .403 & .405 & .407 \\
\hline 680 & .409 & .412 & .414 & .416 & .418 & .420 & .422 & .424 & .426 & $\cdot 428$ \\
\hline 690 & .430 & .433 & .435 & $\cdot 437$ & .439 & .441 & .443 & .445 & .447 & .449 \\
\hline 700 & $9 \cdot 451$ & $9 \cdot 453$ & $9 \cdot 455$ & $9 \cdot 457$ & $9 \cdot 459$ & $9 \cdot 461$ & $9 \cdot 464$ & $9 \cdot 466$ & $9 \cdot 468$ & $9 \cdot 470$ \\
\hline 710 & .472 & .474 & .476 & .478 & .480 & .482 & .484 & .486 & .488 & .490 \\
\hline 720 & .492 & .494 & .496 & .498 & .500 & .502 & .504 & .506 & .508 & .510 \\
\hline 730 & .512 & .514 & .516 & .518 & .520 & .522 & .524 & .526 & .527 & .529 \\
\hline 740 & $\cdot 531$ & $\cdot 533$ & $\cdot 535$ & $\cdot 537$ & .539 & .541 & .543 & .545 & .547 & $\cdot 549$ \\
\hline 750 & $9 \cdot 551$ & $9 \cdot 553$ & 9.555 & $9 \cdot 557$ & $9 \cdot 558$ & $9 \cdot 560$ & $9 \cdot 562$ & $9 \cdot 564$ & $9 \cdot 566$ & 9.568 \\
\hline 760 & $\cdot 570$ & $\cdot 572$ & $\cdot 574$ & $\cdot 576$ & .577 & .579 & $\cdot 581$ & $\cdot 583$ & .585 & $\cdot \mathbf{5 8 7}$ \\
\hline 770 & $\cdot 589$ & .591 & .592 & .594 & .596 & .598 & $\cdot 600$ & $\cdot 602$ & $\cdot 604$ & .605 \\
\hline 780 & 607 & .609 & .611 & .613 & .615 & .617 & .618 & .620 & .622 & .624 \\
\hline 790 & .626 & .628 & .629 & .631 & 633 & .635 & $\cdot 637$ & .638 & .640 & $\cdot 642$ \\
\hline 800 & $9 \cdot 644$ & $9 \cdot 646$ & $9 \cdot 647$ & $9 \cdot 649$ & $9 \cdot 651$ & $9 \cdot 653$ & $9 \cdot 655$ & $9 \cdot 656$ & $9 \cdot 658$ & $9 \cdot 660$ \\
\hline 810 & .662 & .664 & $\cdot 665$ & $\cdot 667$ & $\cdot 669$ & $\cdot 671$ & $\cdot 672$ & $\cdot 674$ & $\cdot 676$ & $\cdot 678$ \\
\hline 820 & $\cdot 679$ & .681 & .683 & .685 & .687 & .688 & .690 & .692 & .693 & .695 \\
\hline 830 & .697 & .699 & .700 & .702 & .704 & .706 & .707 & $\cdot 709$ & .711 & .713 \\
\hline 840 & $\cdot 714$ & .716 & .718 & .719 & .721 & .723 & .725 & .726 & .728 & .730 \\
\hline 850 & $9 \cdot 731$ & $9 \cdot 733$ & $9 \cdot 735$ & $9 \cdot 736$ & 9.738 & $9 \cdot 740$ & $9 \cdot 741$ & $9 \cdot 743$ & $9 \cdot 745$ & $9 \cdot 747$ \\
\hline 860 & .748 & .750 & .752 & .753 & $\cdot 755$ & $\cdot 757$ & $\cdot 758$ & $\cdot 760$ & $\cdot 762$ & $\cdot 763$ \\
\hline 870 & .765 & .767 & .768 & .770 & .771 & .773 & .775 & .776 & .778 & .780 \\
\hline 880 & .781 & .783 & .785 & .786 & .788 & .790 & .791 & .793 & .794 & .796 \\
\hline 890 & .798 & .799 & .801 & .803 & .804 & .806 & $\cdot 807$ & .809 & $\cdot 811$ & .812 \\
\hline 900 & $9 \cdot 814$ & $9 \cdot 815$ & $9 \cdot 817$ & 9.819 & 9.820 & $9 \cdot 822$ & $9 \cdot 823$ & $9 \cdot 825$ & $9 \cdot 827$ & $9 \cdot 828$ \\
\hline 910 & $\cdot 830$ & .831 & $\cdot 833$ & $\cdot \mathbf{8 3 4}$ & $\cdot 836$ & $\cdot \mathbf{8 3 8}$ & $\cdot \mathbf{8 3 9}$ & $\cdot 841$ & $\cdot 842$ & $\cdot 844$ \\
\hline 920 & .845 & .847 & .849 & $\cdot 850$ & .852 & $\cdot 853$ & $\cdot 855$ & .856 & .858 & .860 \\
\hline 930 & .861 & .863 & .864 & .866 & .867 & $\cdot 869$ & $\cdot 870$ & .872 & .873 & $\cdot 875$ \\
\hline 940 & .877 & .878 & .880 & $\cdot 881$ & $\cdot 883$ & .884 & .886 & $\cdot 887$ & .889 & 890 \\
\hline 950 & $9 \cdot 892$ & $9 \cdot 893$ & $9 \cdot 895$ & $9 \cdot 896$ & 9.898 & $9 \cdot 899$ & $\mathbf{9} \cdot 901$ & 9.902 & $9 \cdot 904$ & $9 \cdot 905$ \\
\hline 960 & .907 & .908 & .910 & .911 & .913 & .914 & .916 & .917 & .919 & .920 \\
\hline 970 & .922 & .923 & .925 & .926 & .928 & .929 & .931 & .932 & .934 & .935 \\
\hline 980 & .937 & .938 & .940 & .941 & .943 & .944 & .945 & .947 & .948 & .950 \\
\hline \multirow[t]{3}{*}{990} & .951 & .953 & .954 & .956 & .957 & .959 & .960 & .961 & .963 & .964 \\
\hline & 10 & $10^{2}$ & $10^{3}$ & $10^{4}$ & $10^{5}$ & $10^{\circ}$ & $10^{7}$ & $10^{8}$ & $10^{9}$ & $10^{10}$ \\
\hline & $3 \cdot 322$ & $6 \cdot 644$ & $9 \cdot 966$ & $13 \cdot 288$ & $16 \cdot 610$ & 19.932 & $23 \cdot 253$ & $26 \cdot 575$ & $29 \cdot 897$ & 33.219 \\
\hline
\end{tabular}

